\title{
Quel protocole pour valider ses méthodes : NF T90-210 ou NF V03-110?
}

\author{
Boris GEYNET
}

Centre Technologique Méditerranéen de Métrologie (CT2M), Centre des creusets, Route de Lançon, 13250 Saint-Chamas

\begin{abstract}
.
Accredited laboratories following the ISO 17025 have to validate their analytical methods. Two main standards can be used by laboratories: the NF standard T90-210 and the NF standard V03-110. These two standards were recently revised (respectively in 2009 and 2010) and have both sudden of profound evolutions.
\end{abstract}

Some evolutions are common to both reference standards such as the fact of :

- to work on the found quantity (for example the concentration) rather than the values of information (as for example peak area or absorbance).

- to realize the trial in conditions of intermediate precision rather than in conditions of repeatability.

- to introduce a new more global approach based on the accuracy that is to take into account at the same time the bias and the precision of the method to conclude on the acceptability of the method.

There are nevertheless differences, the most important of which is certainly the determination of the Limit of Quantification (LQ). This LQ is generally very important for laboratories because it is going to allow determining the low limit from which they can give a result with a defined accuracy.

The objective of the paper is to show the points of convergence as well as the main differences between 2 standards to help laboratories in the choice of one of both standards for their methods validation.

\section{Résumé.}

Les laboratoires accrédités selon l'ISO 17025 doivent valider leurs méthodes d'essais non normalisées. Deux principaux référentiels traitent de la validation des méthodes : la norme NF T90-210 et la norme NF V03-110. Ces deux protocoles ont été révisés récemment (respectivement en 2009 et 2010) et ont tous deux subi de profondes évolutions.

Certaines évolutions sont communes aux deux référentiels telles que le fait de :

- travailler sur les grandeurs retrouvées (par exemple la concentration) plutôt que les valeurs d'informations (par exemple une aire de pic ou une absorbance),

- réaliser les essais dans des conditions de fidélité intermédiaire plutôt que dans des conditions de répétabilité,

- introduire une nouvelle approche plus globale basée sur l'exactitude qui prend en compte à la fois la justesse et la fidélité de la méthode pour conclure sur son acceptabilité par rapport au besoin.

Il existe néanmoins des différences dont l'approche méthodologique et la détermination de la Limite de Quantification (LQ). Cette LQ est généralement très importante pour les laboratoires car elle va permettre de déterminer la limite basse à partir de laquelle ils pourront rendre un résultat avec une exactitude définie.

L'objectif de cet article est de présenter les points de convergence ainsi que les principales différences entre les 2 normes afin d'aider les laboratoires dans le choix du protocole à mettre en œuvre pour la validation de leurs méthodes. 


\section{INTRODUCTION}

D'après la norme NF EN ISO CEI 17025 [1], la validation d'une méthode est la «confirmation par examen et l'apport de preuves objectives du fait que les prescriptions particulières en vue d'une utilisation prévue déterminée sont remplies ». Cette étape aboutissant à la déclaration d'aptitude de la méthode se positionne entre son développement et sa mise en routine.

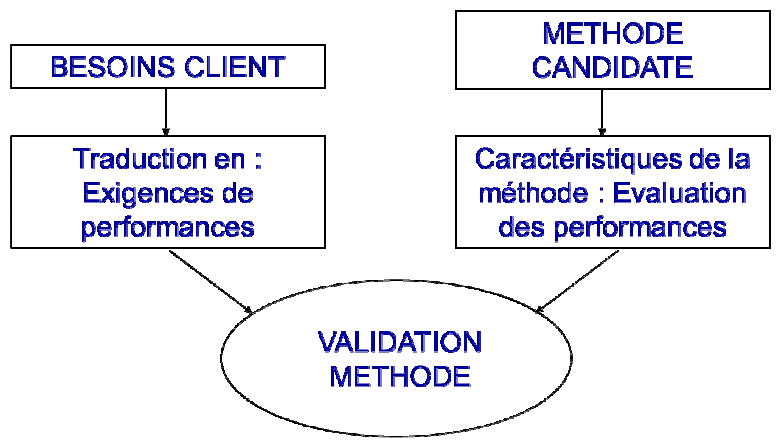

Fig. 1 Définition d'une validation de méthode

Les laboratoires se lançant dans la validation de leurs méthodes d'analyse, d'essai ou d'étalonnage se confrontent systématiquement au choix du protocole de validation. Il n'y a pas de protocole «obligatoire », l'exigence de l'ISO 17025 sur ce point porte sur la nécessité de "valider" sans préciser les moyens d'arriver à la déclaration d'aptitude de la méthode.

Plusieurs normes présentent des protocoles de validation de méthode, ils sont spécifiques à certains domaines d'analyse et ne peuvent pas toujours être appliqués dans leur totalité. C'est donc au laboratoire d'évaluer luimême son besoin en termes de :

- critères de performance à évaluer

- limites acceptables

- méthodologie à adopter

Les deux protocoles les plus couramment utilisés par les laboratoires sont les normes NF T90-210 et la norme NF V03-110. Cet article présente chacune de ces deux normes dans une première partie, puis les similitudes et différences les plus significatives seront mises en évidence.

\section{Présentation des deux protocoles de validation}

\subsection{Le protocole NF T90-210}

Ce document a été rédigé pour la validation des méthodes d'analyse physico-chimiques appliquées au domaine de l'eau. L'application de ce protocole intervient une fois la méthode développée afin de vérifier les différents critères de performance suivants dans des conditions de fidélité intermédiaire :

- la fonction d'étalonnage

- la limite de quantification

- l'influence de la préparation des échantillons

- l'exactitude de la méthode

\begin{tabular}{|c|c|c|}
\hline & $\begin{array}{c}\text { Méthode } \\
\text { développée ou } \\
\text { adaptée }\end{array}$ & $\begin{array}{c}\text { Méthode } \\
\text { normalisée }\end{array}$ \\
\hline $\begin{array}{c}\text { Fonction } \\
\text { d'étalonnage }\end{array}$ & OUI & OUI \\
\hline $\begin{array}{c}\text { Limite de } \\
\text { Quantification }\end{array}$ & OUI & OUI \\
\hline Rendements & OUI & OUI \\
\hline Interférences & OUI & NON \\
\hline Exactitude & OUI & NON \\
\hline
\end{tabular}

Fig. 2 Critères à valider selon la NF T90-210

Le plan d'expérience de type A permet d'évaluer une fonction d'étalonnage dans un domaine de mesure par un test d'adéquation ou une comparaison. La validation de la fonction d'étalonnage peut donc se faire de deux façons :

- soit en comparant les erreurs de modèle observées à des Erreurs Maximales Admissibles (approche métrologique),

- soit en comparant les erreurs de modèle aux erreurs expérimentales (approche statistique).

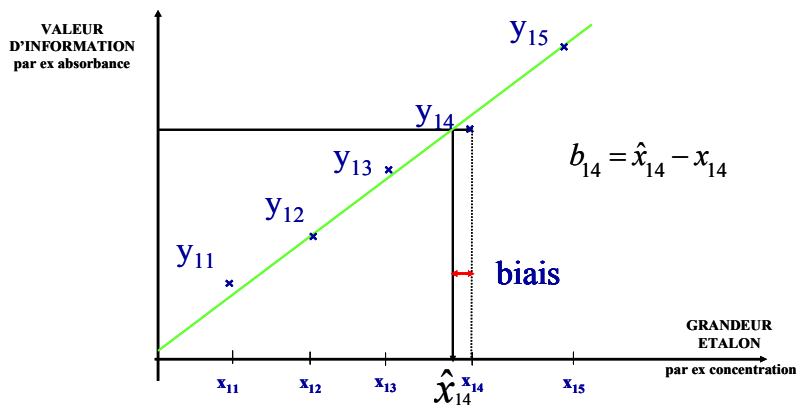

Fig. 3 Représentation des biais liés à la modélisation pour une fonction d'étalonnage linéaire

La limite de quantification doit ensuite être validée en appliquant un plan d'expérience propre. La LQ est définie comme la «plus petite grandeur d'un analyte à 
examiner dans un échantillon pouvant être déterminée quantitativement dans des conditions expérimentales décrites dans la méthode avec une exactitude définie ». La norme NF T90-210 propose la vérification d'une limite de quantification présupposée en étudiant la justesse et la fidélité au niveau de cette valeur.

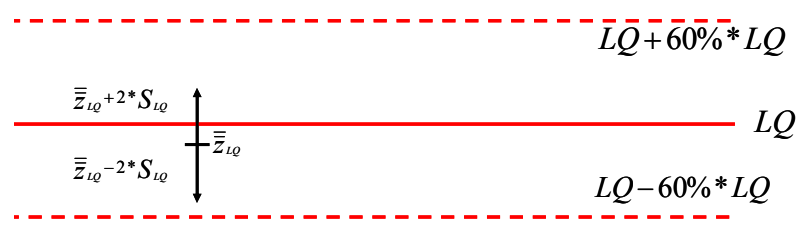

Fig 4 : Validation d'une LQ présupposée

On vérifiera l'exactitude de la méthode à la limite de quantification choisie par rapport à un écart maximal acceptable de $60 \%$.

Le troisième plan d'expérience proposé consiste à déterminer le rendement de la méthode, l'objectif étant de caractériser l'influence de l'étape de préparation lorsque celle-ci n'est pas prise en compte dans l'étude de la fonction d'étalonnage.
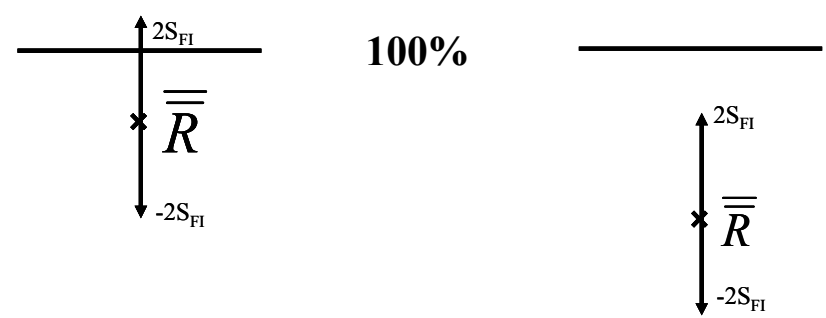

Fig 5 : Deux résultats possibles de l'étude du rendement

L'étude du rendement peut aboutir à deux résultats distincts :

- le rendement moyen n'est pas significativement différent de $100 \%$ (cas de gauche sur la figure 5), le biais n'est donc pas significatif et en règle générale, le laboratoire n'apporte pas de correction de rendement.

- le rendement moyen est significativement différent de 100\% (cas de droite sur la figure 5), une erreur systématique est donc présente. Le laboratoire pourra alors définir une correction de rendement à appliquer dans l'expression du résultat final. Une étude $\mathrm{du}$ rendement sur toute la plage de concentration peut être nécessaire pour mieux connaître la correction de rendement à appliquer. Le laboratoire peut également décider de ne pas corriger ses résultats, il devra alors tenir compte de l'erreur systématique dans son calcul d'incertitude. Cette option induira forcément une incertitude de mesure plus importante.

Les interférences possibles doivent également être étudiées. Les essais seront organisés de façons différentes selon s'il s'agit d'interférences spécifiques (interférent agissant directement sur la réponse de l'analyte) ou non spécifiques (interférent lié à la présence d'un composé qui produit un signal y compris en absence de l'analyte).
Ces deux types d'interférences pourront être étudiés et mis en évidence respectivement par des tests d'ajouts dosés et par ajout du composant interférent.

Le dernier critère de performance à valider dans le protocole NF T90-210 est l'exactitude de la méthode au travers de l'étude de la fidélité intermédiaire et du biais. Cette exactitude est vérifiée sur des échantillons dont les valeurs de référence sont connues et sera comparée à un écart maximal acceptable (EMA) issue d'une exigence réglementaire, normative, client ou fixée par le laboratoire.

Une interprétation du biais de la méthode pour chaque niveau sera d'abord réalisée au travers du calcul du nombre En ou de la comparaison du biais de la méthode avec un biais maximum défini par le laboratoire. La validation de l'exactitude pourra être réalisée en comparant le biais moyen pour chaque niveau aux EMA définis. La figure 6 est une représentation graphique de cette interprétation pour un niveau donné.

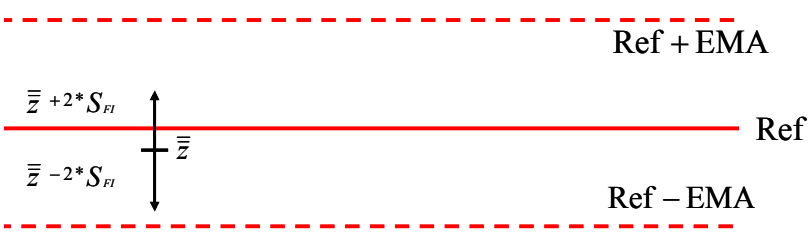

Fig 6 : Validation de l'exactitude d'une méthode

Une fois l'ensemble de ces plans d'expérience réalisés, la déclaration d'aptitude de la méthode va s'appuyer sur les résultats et les interprétations de chacun des critères de performance étudiés.

\subsection{Le protocole NF V03-110}

Ce document propose un protocole de caractérisation en vue de la validation d'une méthode d'analyse quantitative, en particulier dans le domaine agroalimentaire. Son application, tout comme la norme NF T90-210 intervient une fois la méthode développée. La déclaration d'aptitude de la méthode s'appuie ici sur la construction et l'interprétation d'un profil d'exactitude. La norme NF V03-110 nécessite la réalisation d'un plan d'étalonnage et d'un plan de caractérisation qui sont directement liés.

La plan d'étalonnage consiste à établir plusieurs fonctions d'étalonnage avec des solutions étalons et selon la méthode aussi proche que possible de celle réalisée en routine (nombre de niveaux d'étalonnage, de répétitions par niveau et étendue du domaine d'étalonnage). Les précautions à prendre sont le respect de l'indépendance des mesures, le choix des niveaux de solutions étalons régulièrement répartis dans tout le domaine choisi et l'absence de dilutions successives. Les fonctions d'étalonnage sont ensuite représentées graphiquement pour chaque gamme d'étalonnage. 


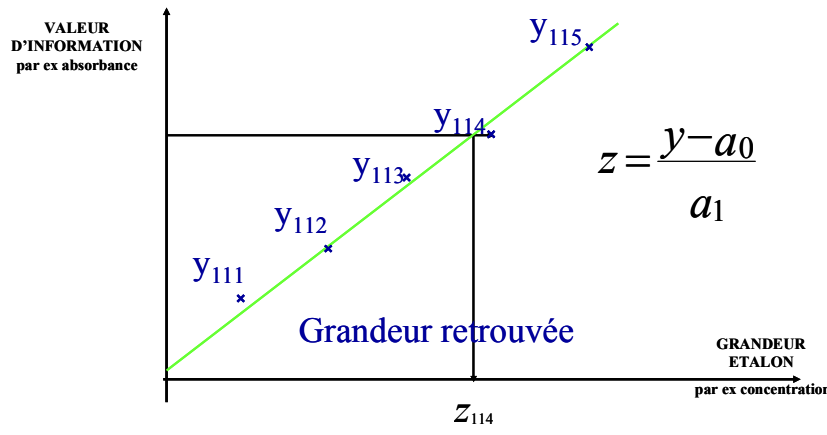

Fig. 7 Représentation des grandeurs retrouvées

Cette étape est recommandée en plus de l'examen visuel des données afin de détecter une erreur flagrante ou des données aberrantes. Les grandeurs retrouvées pourront ensuite être calculées à partir de la fonction d'étalonnage correspondante à chaque gamme.

Le plan de caractérisation permet ensuite de réaliser le profil d'exactitude de la méthode. Pour cela, il est nécessaire d'utiliser des échantillons les plus représentatifs possibles du domaine d'application, stables et homogènes, et dont la concentration est connue le plus exactement possible (MRC, ajouts dosés, échantillons dopés, méthode de référence). Plusieurs séries d'analyses doivent être réalisées dans des conditions de fidélité intermédiaire en respectant les conditions suivantes :

- application du mode opératoire de routine

- indépendance entre les mesures

- utilisation des données d'étalonnage du jour pour calculer les grandeurs retrouvées du plan de caractérisation.

A partir de ces grandeurs retrouvées, plusieurs paramètres pourront être calculés comme le biais absolu, le biais relatif, le taux de récupération ou les limites de tolérance. Le profil d'exactitude est alors établi en positionnant en abscisses les valeurs de référence et en ordonnées le taux de récupération moyen. Pour permettre une interprétation complète, il est nécessaire de placer également sur le graphe les limites de tolérance ainsi que les limites d'acceptabilité définies. Un exemple de profil d'exactitude est présenté sur la figure 8.

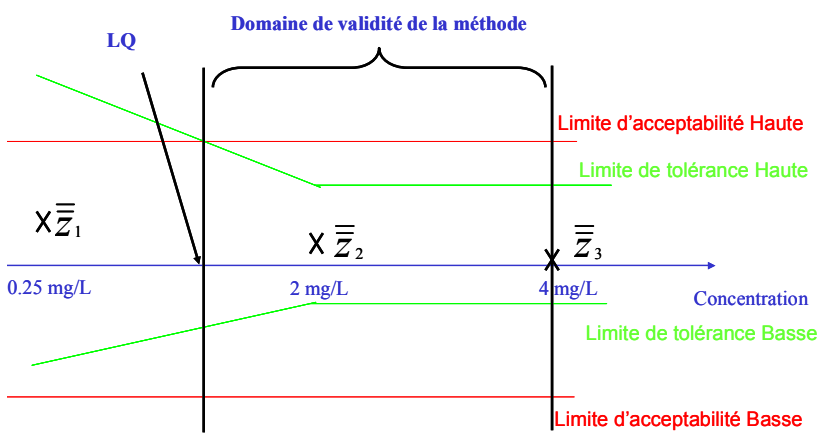

Fig. 8 Exemple de profil d'exactitude

La méthode sera déclarée apte sur un domaine de validité borné. Celui-ci sera défini en vérifiant les deux conditions suivantes :
- le domaine de validité est inclus dans le domaine étudié lors du plan de caractérisation,

- les limites de tolérance calculées sont inférieures aux limites d'acceptabilité définies.

\section{Etude comparative des deux normes}

\subsection{Les principales différences}

Les domaines d'application annoncés dans les deux référentiels constituent une première différence. La norme NF T90-210 s'applique plus particulièrement aux méthodes d'analyse physico-chimiques dans le domaine de l'eau alors que la norme NF V03-110 a été rédigée pour les méthodes d'analyse quantitatives dans le domaine agroalimentaire. L'approche dans la validation des différentes caractéristiques de la méthode est globalement différente. Le protocole NF T90-210 présente plusieurs plans d'expérience permettant de valider les caractéristiques de la méthode une à une, à savoir la fonction d'étalonnage, la limite de quantification, le rendement, les interférences et l'exactitude. 


\begin{tabular}{|c|c|c|}
\hline & NF T90-210 & NF V03-110 \\
\hline 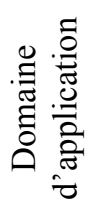 & $\begin{array}{c}\text { Méthodes } \\
\text { d'analyses physico- } \\
\text { chimiques } \\
\text { appliquées au } \\
\text { domaine de l'eau }\end{array}$ & $\begin{array}{c}\text { Méthodes d'analyses } \\
\text { quantitatives, en } \\
\text { particulier dans le } \\
\text { domaine } \\
\text { agroalimentaire }\end{array}$ \\
\hline 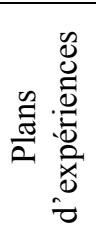 & $\begin{array}{c}\text { Fonction } \\
\text { d'étalonnage } \\
\text { Limite de } \\
\text { quantification } \\
\text { Rendements } \\
\text { Exactitude }\end{array}$ & $\begin{array}{c}\text { Plan d'étalonnage } \\
\text { Plan de } \\
\text { caractérisation }\end{array}$ \\
\hline 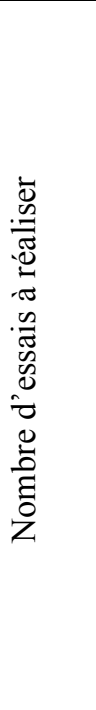 & $\begin{array}{c}\geq 25 \text { pour la } \\
\text { fonction } \\
\text { d'étalonnage } \\
\text { (5 séries, } 5 \text { niveaux) } \\
\geq 10 \text { pour la LQ } \\
\text { présupposée } \\
\text { (5 séries, } 2 \\
\text { répétitions) } \\
\geq 20 \text { pour les } \\
\text { rendements } \\
\text { (5 séries, } 2 \text { niveaux, } \\
2 \text { répétitions) } \\
\geq 30 \text { pour } \\
\text { l'exactitude } \\
\text { (5 séries, } 3 \text { niveaux, } \\
2 \text { répétitions) }\end{array}$ & $\begin{array}{c}\geq 30 \text { pour le plan } \\
\text { d'étalonnage } \\
\text { (5 séries, } 3 \text { niveaux, } \\
2 \text { répétitions) } \\
\geq 30 \text { pour le plan de } \\
\text { caractérisation } \\
\text { (5 séries, } 3 \text { niveaux, } \\
2 \text { répétitions) }\end{array}$ \\
\hline 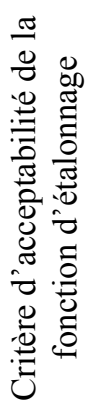 & $\begin{array}{l}\text { Vérification par } \\
\text { rapport à un } \\
\text { «EMA } \\
\text { étalonnage » pour } \\
\text { chaque niveau } \\
\text { OU } \\
\text { Approche } \\
\text { statistique globale } \\
\text { avec test statistique } \\
\text { d'adéquation }\end{array}$ & Aucun \\
\hline 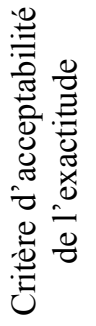 & $\begin{array}{l}\text { Vérification par } \\
\text { rapport à un « EMA } \\
\text { exactitude » pour } \\
\text { chaque niveau } \\
\overline{\bar{z}}+2 S_{F I} \leq \text { ref }+E M A \\
\overline{\bar{z}}-2 S_{F I} \leq \text { ref }-E M A\end{array}$ & $\begin{array}{l}\text { Vérification par } \\
\text { rapport à des limites } \\
\text { d'acceptabilité } \lambda \\
\text { pour chaque niveau } \\
\quad \bar{z}+k_{t o l} S_{I T} \leq \text { ref }+\lambda \\
=\bar{z}-k_{t o l} S_{I T} \leq \text { ref }-\lambda\end{array}$ \\
\hline 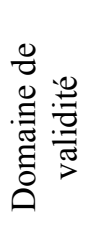 & $\begin{array}{c}\text { De la LQ } \\
\text { présupposée validée } \\
\text { jusqu'à niveau } \\
\text { maximum auquel } \\
\text { l'exactitude a été } \\
\text { validée }\end{array}$ & $\begin{array}{c}\text { Domaine sur lequel } \\
\text { l'intervalle de } \\
\text { tolérance est } \\
\text { compris dans } \\
\text { l'intervalle } \\
\text { d'acceptabilité }\end{array}$ \\
\hline
\end{tabular}

Fig. 9 Tableau comparatif entre les deux normes
Quant à elle, la norme NF V03-110 présente une approche plus globale avec la construction d'un profil d'exactitude, permettant ainsi de valider les différentes caractéristiques à partir d'une seule représentation graphique. Enfin, la façon de déterminer la limite de quantification (LQ) diffère également. Alors que le protocole NF V03-110 décrit comment déterminer expérimentalement la LQ à partir du profil d'exactitude, la norme NF T90-210 préconise la validation expérimentale d'une LQ présupposée qui doit être déterminée avant la réalisation des essais.

\subsection{Les similitudes entre les deux protocoles?}

Malgré les différences citées précédemment, il existe de nombreuses similitudes entre les deux référentiels. Le traitement statistique des résultats se fait à partir des grandeurs retrouvées (exemple : concentration) et non à partir des valeurs d'informations (exemples : aire de pic, absorbance) comme cela a pu être le cas dans les versions précédentes. Les analyses demandées par les plans d'expériences sont systématiquement réalisées dans des conditions de fidélité intermédiaire. Le nombre total de mesures à réaliser pour constituer le dossier de validation de méthode est quasiment identique pour les deux référentiels. Enfin, même si les deux normes ont été établies initialement pour des domaines différents (eau et agroalimentaire), elles sont toutefois applicables à toute méthode d'analyse quantitative.

\section{Conclusion}

Les normes NF T90-210 et NF V03-110 permettent d'atteindre le même objectif (validation d'une méthode) par des moyens différents. Les deux protocoles présentent chacun des avantages et des inconvénients, les laboratoires devront choisir entre deux approches distinctes : la première permet de connaître et valider indépendamment différents critères de performances sur la base de plusieurs plans d'expérience (NF T90-210), la deuxième permet de conclure de façon globale à partir de l'étude de l'exactitude de la méthode (NF V03-110). Le nombre d'essais à réaliser pour l'application des deux normes étant très proches, le coût de la validation sera quasiment identique. Le domaine d'activité du laboratoire et les différences entre les deux approches présentées dans cet article sont des paramètres qui devront être pris en compte dans le choix du protocole de validation à appliquer par les laboratoires. 


\section{Références}

[1] NF EN ISO CEI 17025 - Exigences particulières concernant la compétence des laboratoires d'étalonnages et d'essais.

[2] NF T90-210 version 2009 - Protocole d'évaluation initiale des performances d'une méthode dans un laboratoire.
[3] NF V03-110 version 2010 - Analyse des produits agricoles et alimentaires - Protocole de caractérisation en vue de la validation d'une méthode d'analyse

\section{Partenariat}

Cette publication a bénéficié du soutien financier de l'Union Européenne via le FEDER. 